\title{
Определение способности пористых метакрилатных полимеров к межмолекулярным взаимодействиям с помощью уравнения линейного разложения газохроматографических параметров
}

\author{
Платонова Н.П., Боровикова С.А., Шарапов В.М., Буряк А.К. \\ Институт физической химии и электрохимии им. А.Н. Фрумкина РАН, Москва
}

Поступила в редакцию 11.10.2018 г.

DOI: https://doi.org/10.17308/sorpchrom.2018.18/614

Предложено модифицированное уравнение линейного разложения газохроматографических параметров для описания способности метакрилатных полимеров к различным типам межмолекулярных взаимодействий (ММВ). Уравнение позволяет не только оценивать полярность полимеров, но и определять вклад каждого типа ММВ в удерживание молекул.

Ключевые слова: газовая хроматография, межмолекулярные взаимодействия, полярность сорбентов.

\section{Determination of porous methacrylate polymers ability to solute-sorbent interactions by linear regression of gas-chromatography parameters.}

\author{
Platonova N.P., Borovikova S.A., Sharapov V.M., Buryak A.K. \\ Frumkin Institute of Physical Chemistry RAN, Moscow
}

The linear regression equation of gas chromatography parameters was suggested previously. Each term of this equation characterized sorbents ability to certain types of sorbate-sorbent interactions (SSI). The analysis of the retention data for different molecules by methacrylate polymers shows limitations of the suggested equation. The analysis of the dependence of the retention on polarizability of sorbate molecules allows us to distinguish 4 types of the investigated polymers which differ in quality and polarity of functional groups. For the first type of polymers the linear dependence of retention parameters on polarizability is observed. The polymers with non-polar or with a little amount of weak-polar groups are also of this type. Polymers with middle-polar functional groups and the functional groups containing different amount of nitrogen are of the second and third type. For these polymers the deviation of the retention parameters for methanol from linear dependence on polarizability is observed. For polymers of the fourth type with hardly polar groups the deviation of retention parameters from linearity is observed not only for methanol, but also for the first members of the homologous series of alcohols, ketones and nitrocompounds. In this case the linear regression equation is incorrect. It can probably be caused by the following factors: 1-not all types of SSI are described with suggested equation; 2-physical-chemical parameters of sorbed compounds differ from molecular physical-chemical constants. A modified equation of the linear regression equation of gas chromatography parameters is suggested for describing the ability of methacrylate polymers to different types of SSI. The term describing $\pi$-complexation was advanced to determine the sorbent interaction with $\pi$-electrons of sorbate molecules. The equation allows us not only to estimate polarity of polymers, but also to determine the contribution of different types of SSI in the retention of molecules and thus characterizing the surface chemistry.

Keywords: gas chromatography, solute-sorbent interactions, the sorbents polarity. 


\section{Введение}

В настоящее время большое внимание привлекают расчёты величины хроматографического удерживания на основе свойств сорбента и сорбата. Такие расчёты выполнены для сорбентов с хорошо известной структурой, например, графитированной термической сажи и цеолитов [1,2]. Для сорбентов с неохарактеризованной структурой возможны только приближённые вычисления, например, для пористых полимеров или для неподвижных жидких фаз $[3,4]$. Для сложных органических сорбентов подходы могут быть основаны на теории «обобщенных зарядов» [4] и на эмпирических подходах, состоящих в линейном разложении параметров, составляющих хроматографические величины [3,5-6].

Сорбционные свойства твердого тела определяются его способностью к различным типам межмолекулярных взаимодействий (MМB). Ранее было предложено уравнение линейного разложения газохроматографических параметров (ЛРГП), которое позволяло с удовлетворительной степенью точности оценивать полярность сорбентов и описывать способность некоторых пористых полимеров к тем или иным типам ММВ [5-7]. Дальнейшее накопление экспериментальных данных и анализ результатов описания удерживания с помощью уравнения ЛРГП для широкого круга метакрилатных полимеров выявили ограниченность применимости этой модификации уравнения.

\section{Эксперимент}

Анализ зависимостей удерживания веществ от поляризуемости их молекул и анализ результатов описания удерживания с помощью уравнения ЛРГП для широкого круга метакрилатных полимеров позволили разделить исследованные полимеры на 4 группы, различающиеся количеством и полярностью функциональных групп. Для первой группы полимеров наблюдается линейная зависимость параметров удерживания (свободной энергии или индексов удерживания) органических веществ различных классов от поляризуемости - $\alpha$ их молекул, причем наклон этих зависимостей для различных гомологических рядов одинаков. К этой группе относятся полимеры не содержащие или содержащие небольшое количество слабополярных групп. Для полимеров второй и третьей групп наблюдается отклонение от линейных зависимостей логарифма удерживаемого объема от поляризуемости для первого члена ряда спиртов-метанола, наклон зависимостей различных гомологических рядов одинаков, либо незначительно изменяется. Во вторую и третью группу входят полимеры, содержащие группы средней полярности и различающиеся концентрацией азота. В этих случаях уравнение ЛРГП описывает удерживание с различной степенью точности. Для полимеров четвертой группы, содержащих полярные группы, например, катионы металлов, отклонение от линейности наблюдаются не только для метанола, но и для первых членов гомологических рядов других полярных молекул (кетонов, нитросоединений) и в этом случае уравнение ЛРГП неприменимо. Причинами неудовлетворительного описания удерживания предложенным уравнением могут быть следующие факторы: 1) не все возможные типы ММВ учитываются предложенным уравнением. 2) физико-химические параметры сорбированных веществ отличаются от молекулярных физико-химических констант.

Предложенное уравнение не описывает удерживание ароматических соединений, потому что оно не учитывает специфики взаимодействий с $\pi$-электронами. Мы предположили, что энергия этого взаимодействия пропорциональна числу $\pi$ электронов и обратно пропорциональна объему молекул и ввели в уравнение сла- 
гаемое, оценивающее $\pi$-комплексообразование (формула 1). Затем были выбраны тестовые вещества с более представительным набором функциональных групп, способные к различным типам MМВ и имеющие наиболее различающиеся физикохимические параметры молекул (поляризуемость, дипольный момент, донорные и акцепторные константы). Для проверки корректности набора тестовых веществ составлялись различные группы веществ разных классов, проводился расчет коэффициентов уравнения ЛРГП и оценивалось ошибка расчета (табл. 1). Как видно из приведенной таблицы, исключение из стандартного набора какого-либо класса веществ проводят к неприменимости уравнения (ошибка расчета достигает 30\%). Исключение из набора веществ пиридина увеличивает ошибку расчета незначительно, но сильно сказывается на величине акцепторной способности поверхности. Также нежелательно при расчете использовать гексан вместо нонана, что приводит к уменьшению коэффициента дисперсионного взаимодействия. Замена метанола на его гомологи допустима.

Таблица 1. Оценка корректности набора тестовых веществ. Стандарт-нонан, $\mathrm{MeOH}$, $\mathrm{BuOH}, \mathrm{Pr}_{2} \mathrm{O}$, ацетон, EtAc, $\mathrm{MeNO}_{2}, \mathrm{MeCN}, \mathrm{Pyr}, \mathrm{Bz}, \mathrm{MePh}$

\begin{tabular}{|c|c|c|c|c|c|c|}
\hline & $\begin{array}{c}\text { Ошибка } \\
\text { расчета, \% }\end{array}$ & К1 & К2 & К3 & К4 & К5 \\
\hline Стандарт & 6.4 & 110 & 61 & 105 & 26 & 158 \\
\hline -Acetone & 31.2 & & & & & \\
\hline- -EtAc & 30.6 & & & & & \\
\hline$-\mathrm{MeNO} 2$ & 32.2 & & & & & \\
\hline$-\mathrm{MeCN}$ & 27.5 & & & & & \\
\hline$-\mathrm{MePh}$ & 7.0 & 111 & 61 & 104 & 27 & 136 \\
\hline$-\mathrm{Pyr}$ & 7.2 & 101 & 61 & 120 & 12 & 125 \\
\hline $\mathrm{C} 6(\mathrm{C} 9)$ & 6.0 & 90 & 55 & 109 & 25 & 208 \\
\hline EtOH(MeOH) & 5.6 & 111 & 60 & 97 & 25 & 148 \\
\hline PrOH(MeOH) & 5.4 & 112 & 61 & 93 & 25 & 145 \\
\hline
\end{tabular}

Таким образом, уравнение ЛРГП имеет вид (формула 1):

$$
\mathrm{E}_{\text {сорб. }}=\mathrm{K}_{1} \alpha \mathrm{G}+\mathrm{K}_{2}\left(2 \alpha^{2} / 3 \mathrm{kT}+\alpha \mathrm{G}\right)+\mathrm{K}_{3} \mathrm{~W}_{\mathrm{a}}+\mathrm{K}_{4} \mathrm{~W}_{\mathrm{d}}+\mathrm{K}_{5} \mathrm{n}_{\pi} / \mathrm{v}+\mathrm{K}_{6}
$$

где $K_{1-5}$-коэффициенты, пропорциональные физико-химическим характеристикам сорбента, входящим в выражение для каждого типа МMB; $\mathrm{K}_{6}$ - свободный член, включающий неучтенные $\mathrm{MMB} ; \alpha$ - поляризуемость сорбируемой молекулы, $\mathrm{A}^{\text {o }}$; $\mu$-ее дипольный момент, $\mathrm{D} ; \mathrm{W}_{\mathrm{a}}, \mathrm{W}_{\mathrm{d}}$ - акцепторная и донорная энергетическая константа, $(\mathrm{kJ} / \mathrm{mol})^{1 / 2} ; \mathrm{G}$ - стерический фактор, отражающий неполноту контакта сорбирующейся молекулы с поверхностью сорбента; n- число $\pi$-электронов в сорбирующейся молекуле; v- мольный объем, $\mathrm{cm}^{3}$.

\section{Обсуждение результатов}

По предложенному уравнению были обработаны данные для модифицированных метакрилатных полимеров, полученных на основе сополимеров 2,3эпоксипропилметакрилата (2,3ЭПМА) с этилендиметакрилатом (ЭДМА) с различной степенью сшивки. Модифицирование осуществлялось ароматическими соединениями, аминами, аминоспиртами, нитрилами и сложными эфирами. Были также обработаны данные для сополимеров 2,3ЭПМА, стирола и ЭДМА $[3,8,9]$ с различным содержанием стирола. Из таблицы 2 видно, что полярность фенилсодержащих полимеров понижена по сравнению с исходным сополимером, особенно, если фенильные группы находятся в основной цепи полимера (обp. gst10 и gst50) за счет 
уменьшения акцепторной способности, несмотря на некоторое увеличение донорной способности поверхности. Полярность понижается тем сильнее, чем больше стирола в полимере (обр. gst10 и gst50). Аминированные полимеры полярнее исходного вследствие значительно возросшей донорной способностей поверхности. Сравнивая образцы с близким содержанием азота (обр. g60am, g60deta и g60 $\mathrm{C}_{12}$ ), можно видеть, что полярность полимеров не коррелирует с содержанием азота и не отражает изменение природы функциональных групп. В то же время коэффициенты уравнения ЛРГП заметно изменяются в зависимости от природы функциональных групп. Т.е. полярность менее чувствительная к химии поверхности характеристика, чем коэффициенты уравнения, характеризующие способность поверхности к различным типам взаимодействий. Для полимеров, модифицированных одним тем же амином коэффициенты $K_{1}$ увеличиваются с увеличением степени сшивки, т.к. при этом увеличивается удельная поверхность образцов, а коэффициенты ориентационного и донорного взаимодействий $\mathrm{K}_{2}$ и $\mathrm{K}_{3}$ уменьшаются т.к. при этом уменьшается содержание азота в образцах (рис.1). Акцепторная способность поверхности К 4 незначительно возрастает.

Таблица 2. Коэффициенты уравнения ЛРГП и полярность модифицированных метакрилатных полимеров

\begin{tabular}{|c|c|c|c|c|c|c|c|c|c|}
\hline Образец & Функц. группы & $\mathrm{S}_{\mathrm{y} \text {. }}$ & $\begin{array}{l}\mathrm{N}, \\
\%\end{array}$ & K1 & K2 & K3 & K4 & K5 & $\mathrm{P}$ \\
\hline g60 & $-\mathrm{O}-$ & 51 & 0 & 133 & 50 & 63 & 24 & 419 & 1.99 \\
\hline $\mathrm{g} 60 \mathrm{Bz}$ & & 60 & 0 & 130 & 55 & 80 & 5 & 295 & 1.70 \\
\hline Gst10 & & 53 & 0 & 125 & 47 & 73 & 10 & 283 & 1.60 \\
\hline Gst50 & & 50 & 0 & 131 & 42 & 70 & 1 & 341 & 1.46 \\
\hline g60dea & $-\mathrm{OH},-\mathrm{N}=$ & 41 & 3.5 & 108 & 59 & 104 & 16 & 347 & 2.15 \\
\hline g80am & $\begin{array}{c}-\mathrm{OH},-\mathrm{NH}_{2},- \\
\mathrm{NH}-\mathrm{-N}=\end{array}$ & 47 & 3.3 & 113 & 54 & 115 & 63 & 63 & 2.47 \\
\hline g60am & $\begin{array}{c}-\mathrm{OH},-\mathrm{NH}_{2},- \\
\mathrm{NH}-,-\mathrm{N}=\end{array}$ & 81 & 2.7 & 166 & 52 & 119 & 16 & 313 & 2.09 \\
\hline g60deta & $\begin{array}{c}-\mathrm{OH},-\mathrm{NH}_{2},- \\
\mathrm{NH}-,-\mathrm{N}=\end{array}$ & 52 & 7.4 & 110 & 61 & 105 & 26 & 158 & 2.06 \\
\hline $\begin{array}{l}\text { g60deta } \\
\text { alk }\end{array}$ & $-\mathrm{OH},-\mathrm{N}=$ & 43 & 6,0 & 100 & 71 & 143 & 1 & - & 1,89 \\
\hline g40deta & $\begin{array}{c}\mathrm{OH},-\mathrm{NH}_{2},- \\
\mathrm{NH}-,-\mathrm{N}=\end{array}$ & 117 & 5.8 & - & - & - & - & - & - \\
\hline g90deta & $\begin{array}{c}\mathrm{OH},-\mathrm{NH}_{2},- \\
\mathrm{NH}-,-\mathrm{N}=\end{array}$ & 9 & 12 & - & - & - & - & - & - \\
\hline
\end{tabular}

Помимо общей оценки полярности полимеров и их способности к различным типам ММВ представляет интерес рассмотреть вклады каждого типа ММВ в удерживание различных молекул. В табл. 3 приведены рассчитанные по уравнению ЛРГП вклады каждого слагаемого некоторых тестовых веществ на аминированных полимерах в общую энергию сорбции. Для полимеров с различной степенью сшивки, модифицированных одним и тем же аминирующим агентом, при сорбции всех тестовых молекул вклад дисперсионного взаимодействия уменьшается вместе с уменьшением удельной поверхности, а вклад специфического взаимодействия уве- 
личивается в результате увеличения количества аминогрупп. При адсорбции метанола на всех образцах преобладает вклад донорного взаимодействия.

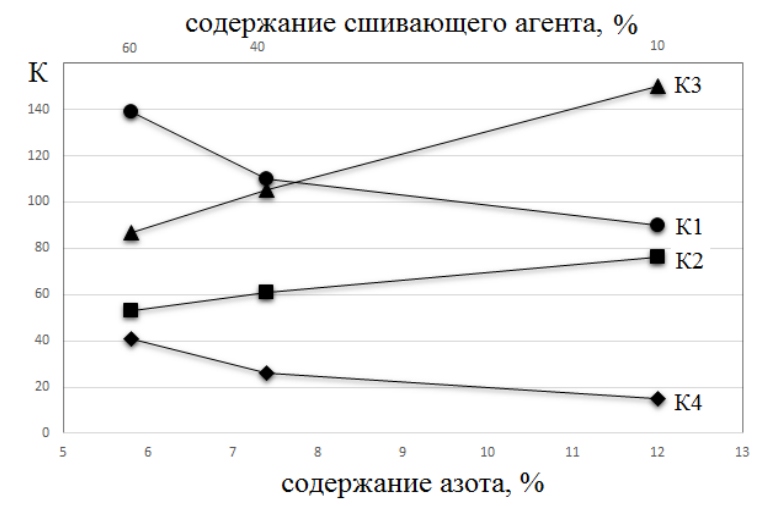

Рис. 1. Зависимость коэффициентов уравнения ЛРГП от содержания азота в образцах gdeta

Таблица 3. Вклады (\%) энергии каждого типа ММВ в общую энергию сорбции молекул

\begin{tabular}{|c|c|c|c|c|c|c|c|c|c|}
\hline Образец & $\mathrm{N}, \%$ & $\mathrm{D}_{\mathrm{i}}$ & $\mathrm{MeOH}$ & $\left(\mathrm{CH}_{3}\right)_{2} \mathrm{CO}$ & EtAc & $\mathrm{MeNO}_{2}$ & $\mathrm{MeCN}$ & Pyr & $\mathrm{Bz}$ \\
\hline \multirow{5}{*}{ g60am } & \multirow{5}{*}{2.7} & $\mathrm{D}_{1}$ & 34 & 68 & 86 & 66 & 51 & 72 & 88 \\
\hline & & $\mathrm{D}_{2}$ & 9 & 24 & 8 & 27 & 42 & 9 & 0 \\
\hline & & $\mathrm{D}_{3}$ & 47 & 0 & 0 & 0 & 0 & 0 & 0 \\
\hline & & $\mathrm{D}_{4}$ & 10 & 8 & 6 & 7 & 7 & 11 & 3 \\
\hline & & $\mathrm{D}_{5}$ & 0 & 0 & 0 & 0 & 0 & 8 & 9 \\
\hline \multirow{5}{*}{ g80am } & \multirow{5}{*}{3.3} & $\mathrm{D}_{1}$ & 24 & 52 & 69 & 52 & 40 & 52 & 84 \\
\hline & & $\mathrm{D}_{2}$ & 8 & 20 & 8 & 24 & 37 & 9 & 0 \\
\hline & & $\mathrm{D}_{3}$ & 37 & 0 & 0 & 0 & 0 & 0 & 0 \\
\hline & & $\mathrm{D}_{4}$ & 31 & 28 & 23 & 24 & 23 & 38 & 14 \\
\hline & & $\mathrm{D}_{5}$ & 0 & 0 & 0 & 0 & 0 & 1 & 2 \\
\hline \multirow{5}{*}{ g60dea } & \multirow{5}{*}{3.5} & $\mathrm{D}_{1}$ & 24 & 52 & 77 & 49 & 34 & 59 & 80 \\
\hline & & $\mathrm{D}_{2}$ & 13 & 37 & 14 & 41 & 58 & 15 & 0 \\
\hline & & $\mathrm{D}_{3}$ & 51 & 0 & 0 & 0 & 0 & 0 & 0 \\
\hline & & $\mathrm{D}_{4}$ & 12 & 11 & 9 & 10 & 8 & 15 & 5 \\
\hline & & $\mathrm{D}_{5}$ & 0 & 0 & 0 & 0 & 0 & 11 & 15 \\
\hline \multirow{5}{*}{ g60deta } & \multirow{5}{*}{7.4} & $\mathrm{D}_{1}$ & 21 & 46 & 71 & 43 & 30 & 56 & 84 \\
\hline & & $\mathrm{D}_{2}$ & 12 & 36 & 14 & 41 & 57 & 15 & 0 \\
\hline & & $\mathrm{D}_{3}$ & 49 & 0 & 0 & 0 & 0 & 0 & 0 \\
\hline & & $\mathrm{D}_{4}$ & 18 & 18 & 15 & 16 & 13 & 24 & 8 \\
\hline & & $\mathrm{D}_{5}$ & 0 & 0 & 0 & 0 & 0 & 5 & 8 \\
\hline \multirow{5}{*}{$\begin{array}{l}\text { g60deta } \\
\text { alk }\end{array}$} & \multirow{5}{*}{6.0} & $\overline{D_{1}}$ & 22 & 50 & 77 & 47 & 32 & 61 & 85 \\
\hline & & $\mathrm{D}_{2}$ & 13 & 42 & 16 & 46 & 63 & 18 & 0 \\
\hline & & $\mathrm{D}_{3}$ & 58 & 0 & 0 & 0 & 0 & 0 & 0 \\
\hline & & $\mathrm{D}_{4}$ & 7 & 8 & 7 & 7 & 5 & 11 & 3 \\
\hline & & $\mathrm{D}_{5}$ & 0 & 0 & 0 & 0 & 0 & 10 & 12 \\
\hline \multirow{5}{*}{ g60teta } & \multirow{5}{*}{8.3} & $\overline{D_{1}}$ & 26 & 57 & 80 & 55 & 39 & 64 & 84 \\
\hline & & $\mathrm{D}_{2}$ & 12 & 35 & 13 & 38 & 55 & 15 & 0 \\
\hline & & $\mathrm{D}_{3}$ & 54 & 0 & 0 & 0 & 0 & 0 & 0 \\
\hline & & $\mathrm{D}_{4}$ & 8 & 8 & 7 & 7 & 6 & 11 & 3 \\
\hline & & $\mathrm{D}_{5}$ & 0 & 0 & 0 & 0 & 0 & 10 & 13 \\
\hline
\end{tabular}

При сорбции нитрометана, ацетона и, особенно, ацетонитрила наиболее значительный вклад вносит ориентационное взаимодействие. Сравнивая образцы с близкими значениями содержания азота и полярности (g80am, g60dea), можно ви- 
деть, что на образце g80am, содержащем первичные и вторичные аминогруппы, увеличивается вклад акцепторного взаимодействия, в случае образца g60dea, содержащем третичные аминогруппы, преобладает ориентационное взаимодействие. Такая же закономерность проявляется на образцах g60deta и g60deta alk. Увеличение основности аминогрупп при их алкилировании (образцы g60deta и g60deta alk) приводит к увеличению вкладов ориентационного взаимодействия, несмотря на уменьшение количества аминогрупп. В этом случае полярность образца определяется основностью аминогрупп, а не их количеством. Таким образом, на полимерах, содержащих высокоосновные третичные аминогруппы значительный вклад вносит ориентационное взаимодействие с сорбирующейся молекулой, полимеры, содержащие первичные и вторичные аминогруппы меньшей основности, проявляют акцепторные свойства поверхности. Рассматривая образец g60deta, можно видеть, что на нем, так же как на образцах с третичными аминогруппами преобладает ориентационное взаимодействие. Полученные результаты позволяют предположить, что при аминировании полимеров диэтиленамином и триэтиленамином на поверхности полимера образуется значительное количество третичных аминогрупп, повидимому, в результате дополнительной сшивки с участием аминогрупп.

\section{Заключение}

Для оценки взаимодействия сорбента с $\pi$-электронами молекулы сорбата в уравнение ЛРГП введено слагаемое, описывающее $\pi$-комплексообразование. По предложенному уравнению обработаны данные для метакрилатных полимеров. Предложенное модифицированное уравнение ЛРГП позволяет не только оценивать полярность полимеров, но и выделять преобладающий тип ММВ при сорбции, что, в свою очередь, несет информацию о химии поверхности сорбента.

\section{Список литературы}

1. Киселев А.В. Межмолекулярные взаимодействия в адсорбции и хроматографии. М. Высшая школа. 1986. 360.

2. Д.Д.Матюшин, А.К.Буряк // Сорбичионные и хроматографические проиессы. 2017. T. 17. №. 2. C. 204-211.

3. Афанасьева О.В., Платонова Н.П., Шарапов А.В., Градил И. // Журн. физ. химии. 1999. Т.73. №11. С. 2048-2052.

4. Долгоносов А.М., Рудаков О.Б., Суровцев И.С., Прудковский А.Г.. Колоночная аналитическая хроматография как объект математического моделирования. Воронеж. Воронежский государственный архитектурно-строительный университет. 2013. 400 с.
5. Larionov O.G., Petrenko V.V., Platonova N.P. // J. Chromatogr. 1991. Vol. 537. pp. 295303.

6. Larionov O.G., Petrenko V.V., Platonova N.P. // J. Chromatogr. 1991. Vol. 552. pp. 3141.

7. Платонова Н.П., Буряк А.К., Ульянов А.В., Блинникова 3.К. и др. // Сорбциинные и хроматографические прочессы. 2012. Т.12. № 4. C. 575-582.

8. Платонова Н.П., Татаурова О.Г., Булгакова Р.А., Градил И. // Журн. физ. химии. 1993. T. 67. № 7. С. 1480-1482.

9. Платонова Н.П., Татаурова О.Г., Градил И. // Журн. физ. химии. 1996. Т. 70. № 9. С. 1688-1691.

\section{References}

1. Kiselev A.V. Mezhmolekulyarnye vzaimodeistviya $\mathrm{v}$ adsorbtsii i chromatographii, M., Vysshaya shkola, 1986, 360.

2. Matyushin D.D., Buryak A.K., Sorbtsionnye $i$ khromatograficheskie protsessy, 2017, Vol. 17, No 2, pp. 204-211. 
3. Afanaseva O.V., Platonova N.P., Sharapov A.V., Hradil J., J. of Phisical Chemistry, 1999, Vol. 73, No 11, pp. 2048-2052.

4. Dolgonosov A.M., Rudakov O.B., Suvorovtsev I.S., Prudkovskii A.G., Kolonotchnaya analititcheskaya cromatographiya kak ob'ekt matematitcheskogo modelirovaniya, Voronezh, Voronezhski gosudarstvennyi architekturnostroitelnyi universitet, 2013, $400 \mathrm{p}$.

5. Larionov O.G., Petrenko V.V., Platonova N.P., J. Chromatogr., 1991, Vol. 537, pp. 295303.

Платонова Наталия Петровна - старший научный сотрудник, кандидат химических наук, Институт физической химии и электрохимии им. А.Н.Фрумкина РАН, Москва

Боровикова Светлана Александровна - научный сотрудник, кандидат химических наук, Институт физической химии и электрохимии им. А.Н.Фрумкина РАН, Москва

Шарапов Валерий Михайлович - ведущий научный сотрудник, доктор физикоматематических наук, Институт физической химии и электрохимии им. А.Н.Фрумкина РАН, Москва

Буряк Алексей Константинович - директор, доктор химических наук, Институт физической химии и электрохимии им. А.Н.Фрумкина РАН, Москва
6. Larionov O.G., Petrenko V.V., Platonova N.P., J. Chromatogr., 1991, Vol. 552, pp. 3141.

7. Platonova N.P., Buryak A.K., Ulyanov A.V., Blinnikova Z.K. et al., Sorbtsionnye $i$ khromatograficheskie protsessy, 2012, Vol. 12, No 4, pp. 575-582.

8. Platonova N.P., Tataurova O.G., Bulgakova R.A., Hradil J., J. of Phisical Chemistry, 1993, Vol. 67, No 7, pp. 1480-1482

9. Platonova N.P., Tataurova O.G., Hradil J., J. of Phisical Chemistry, 1996, Vol. 70, No 9, pp. 1688-1691.

Platonova Nataliya P. - researcher, Ph.D. Frumkin Institute of Phisical and Electrochemistry RAN, Moscow, e-mail: npplatonova@yandex.ru

Borovikova Svetlana A. - researcher, Ph.D. Frumkin Institute of Phisical and Electrochemistry RAN, Moscow

Sharapov Valeri M. - researcher, grand Ph.D. Frumkin Institute of Phisical and Electrochemistry RAN, Moscow

Buryak Alexei K. - director, grand Ph.D. Frumkin Institute of Phisical and Electrochemistry RAN, Moscow 